CERN-TH/96-219

hep-th/9608122

\title{
Non-Integrability of Two-Dimensional QCD
}

\author{
Werner Krauth $^{1}$ and Matthias Staudacher ${ }^{2}$ \\ ${ }^{1}$ CNRS-Laboratoire de Physique Statistique de l'ENS \\ 24, rue Lhomond \\ F-75231 Paris Cedex 05, France \\ ${ }^{2}$ CERN, Theory Division \\ CH-1211 Geneva 23, Switzerland
}

\begin{abstract}
In this paper we numerically demonstrate that massless two-dimensional QCD is not integrable. To this aim, we explicitly solve the 't Hooft integral equation for bound states by an adaptive spline procedure, and compute the decay amplitudes. These amplitudes significantly differ from zero except in all cases in which the decay also produces a pion.
\end{abstract}

CERN-TH/96-219

August 1996

Electronic mail addresses: krauth@physique.ens.fr, matthias@nxth04.cern.ch. 


\section{Introduction and Conclusions}

Integrability imposes very strong constraints on a quantum field theory. The scattering amplitudes become purely elastic and pair production is suppressed. These features are rather unrealistic from the point of view of four-dimensional quantum field theories of elementary particles; and indeed, integrability only exists in two dimensions. There it is however ubiquitous, and allows in many cases the determination of the exact S-matrix.

One of the most interesting two-dimensional model quantum field theories is $Q C D_{2}$. Pure Yang-Mills theory in two dimensions is essentially trivial due to the absence of transverse degrees of freedom; however, adding minimally coupled fermions results in a model which is believed to display some of the realistic features of the four-dimensional theory. Indeed, as 't Hooft [1] first showed in the leading $\frac{1}{N}$ approximation ( $N$ being the order of the gauge group), the model contains a tower of mesons built from quark-antiquark pairs, with nearly linearly rising meson masses. No free quarks are present. This furnishes a simple yet explicit example for confinement.

Recently, it was argued in a series of papers that the model should be integrable in the case of massless bare quarks [2] for any $N$. This conclusion was reached from the study of a bosonized version of the model, in which seemingly an infinite number of conserved charges existed ( $c f[3]$ for a review of this and related work). An ansatz for an exact, factorized S-matrix was proposed in [4].

The assertions contained in the above work are very strong, and clearly warrant verification in a more direct, and controlled fashion. This may be accomplished by going back to 't Hooft's large $N$ limit. Indeed, the large $N$ limit has been a very useful tool to test the S-matrix of integrable vector-like theories like the $O(N)$ model or the $U(N)$ Gross-Neveu model [5]. The crucial idea is to investigate the occurrence of pair production [6].

In the following we carefully compute the decay amplitudes in the chiral 't Hooft model. This requires a numerical solution of 't Hooft's bound state integral equation for which no general analytic solution is known to date. We present in this paper an adaptive spline procedure, which allows to compute numerically exact solutions (both in the massless and the massive case) with negligible effort. The numerical code is made available to the interested reader; it outperforms the algorithms which were previously applied to this problem. The method most frequently used in the past is based on the use of Chebyshev polynomials and was developed in [7].

We definitely find non-zero probabilities for pair production to first order in $\frac{1}{N}$. This 
result renders inconceivable the integrability of chiral two-dimensional $Q C D$ with gauge group $S U(N)$.

In contrast, we establish the complete on-shell decoupling of the massless $N=\infty$ pion. While expected on general grounds, the result displays a mathematically intriguing property of 't Hooft's bound state problem. The decoupling is satisfied to a very high numerical precision, which illustrates the quality of our method.

\section{Decay Amplitudes in the 't Hooft Model}

$Q C D_{2}$ with gauge group $S U(N)$ is described by the Lagrangian

$$
\mathcal{L}=-\frac{N}{4 g^{2}} \operatorname{Tr} F_{\mu \nu} F^{\mu \nu}+\bar{\psi}\left(i \gamma^{\mu} D_{\mu}-m\right) \psi,
$$

where the field strength is $F_{\mu \nu}=\partial_{\mu} A_{\nu}-\partial_{\nu} A_{\mu}+i\left[A_{\mu}, A_{\nu}\right]$ and the covariant derivative is $D_{\mu}=\partial_{\mu}+i A_{\mu}$. The gauge potential $A_{\mu}$ is given by $N \times N$ hermitian traceless matrices. In the light-cone gauge and the limit $N \rightarrow \infty$, 't Hooft has derived the Bethe-Salpeter equation for meson bound states [1]:

$$
\mu^{2} \phi(x)=\frac{\gamma-1}{x(1-x)} \phi(x)-\int_{0}^{1} d y \frac{\mathcal{P}}{(x-y)^{2}} \phi(y)
$$

Here $\phi(x)$ is a light-cone wave function with meson mass-square eigenvalue $\mu^{2}$ and bare quark mass-square $\gamma$. In the chiral limit one has $\gamma=0$. $\mathcal{P}$ denotes principal value integration and all mass-squares are in units of $\frac{g^{2}}{\pi}$.

At $N=\infty$ the mesons are trivially stable since decay processes are suppressed by at least a factor of $\frac{1}{N}$. One therefore needs the amplitude to order $\frac{1}{N}$ for a meson decaying into two less massive mesons. This was first worked out in [8]; the amplitude $\mathcal{A}(\omega)$ is

$$
\mathcal{A}\left(i, f_{1}, f_{2} ; \omega\right)=\frac{1}{1-\omega} \int_{0}^{\omega} d x \phi_{i}(x) \phi_{f_{1}}\left(\frac{x}{\omega}\right) \Phi_{f_{2}}\left(\frac{x-\omega}{1-\omega}\right)-\frac{1}{\omega} \int_{\omega}^{1} d x \phi_{i}(x) \Phi_{f_{1}}\left(\frac{x}{\omega}\right) \phi_{f_{2}}\left(\frac{x-\omega}{1-\omega}\right) .
$$

Here $\phi_{i}, \phi_{f_{1}}, \phi_{f_{2}}$ are the wave functions of the initial and first and second final mesons, respectively. The quark and the antiquark of the initial meson go to the second and first final meson, respectively. The quark-antiquark-meson vertex function $\Phi(x)$ (with $x \notin[0,1])$ is related to the wave function $\phi(x)$ through

$$
\Phi(x)=\int_{0}^{1} d y \frac{1}{(x-y)^{2}} \phi(y),
$$


and the on-shell values of the kinematic parameter $\omega$ are

$$
\omega_{ \pm}=\frac{\mu_{i}^{2}+\mu_{f_{1}}^{2}-\mu_{f_{2}}^{2} \mp \sqrt{\left(\mu_{i}^{2}+\mu_{f_{2}}^{2}-\mu_{f_{1}}^{2}\right)^{2}-4 \mu_{i}^{2} \mu_{f_{2}}^{2}}}{2 \mu_{i}^{2}}
$$

where $\omega_{+}$and $\omega_{-}$correspond to a right moving and left moving final state $f_{1}$. Clearly pair production is only kinematically possible if $\mu_{i} \geq \mu_{f_{1}}+\mu_{f_{2}}$. In the special case of identical final states $f_{1}=f_{2}=f$, eq. (5) simplifies to

$$
\omega_{ \pm}=\frac{1}{2} \mp \frac{1}{2} \sqrt{1-4 \frac{\mu_{f}^{2}}{\mu_{i}^{2}}}
$$

Note that these are partial decay amplitudes, which contain all the dynamical information. To get the full amplitudes entering the decay probabilities we have to add the partial ones. Thus we add the amplitudes for the quark of the initial particle going to the first or second final state. One then finds for the total on-shell amplitude $\mathcal{A}=\left(1-(-1)^{\sigma_{i}+\sigma_{f_{1}}+\sigma_{f_{2}}}\right)\left(\mathcal{A}\left(i, f_{1}, f_{2} ; \omega_{+}\right)+\mathcal{A}\left(i, f_{1}, f_{2} ; \omega_{-}\right)\right)$. Here $\sigma=+1$ or $\sigma=-1$ for a state with even or odd parity. Therefore half of the decays are forbidden due to the parity symmetry. Below we will only consider allowed decays.

\section{The Numerical Method}

The integral equation eq. (21) is too singular to be solved by direct discretization. One is practically forced to introduce a set of smooth test functions, for which the principal value integral in eq. (2) can be computed exactly. Besides being smooth, an ideal test function has to be sufficiently flexible in order not to impose its own analytical structure onto the solution of the equation. Cubic splines (which are also much used in computer graphics) provide an excellent approximation scheme for difficult eigenvalue problems as the present one. The power of this approach is very often overlooked.

Our idea is to search the best solution of the 't Hooft equation among the spline functions $\phi(x)$ on the interval $[0,1]$. The interpolating cubic spline is uniquely defined by its function values $\phi_{i}=\phi\left(x_{i}\right)$ on a set of node points $x_{i}, i=1, N$ with $x_{i}<x_{i+1}$ (and by the derivatives at $x=0$ and at $x=1, c f$ [9]). Standard algorithms [9], [10] allow to compute the function which, on the interval $\left[x_{i}, x_{i+1}\right]$, is a third-order polynomial and which passes smoothly from any interval into the adjacent ones (intervals are patched together in such a way that $\phi$ and its first two derivatives are continuous at $x_{i}$ ).

For a given set of parameters (the values of $\phi_{i}, i=1, \ldots, N$, the derivatives $d \phi /\left.d x\right|_{x=0}$ and

$d \phi /\left.d x\right|_{x=1}$, as well as $\mu^{2}$ ), the eq. (2) will not be satisfied, but we can choose the parameters 
such that the discrepancy between the l.h.s. and the r.h.s. of eq. (2) is minimized. Using a standard conjugate gradient algorithm for minimization in high dimensions, we easily find different sets of parameters which let this discrepancy disappear for all (numerical) intents and purposes. $\mu^{2}$ is one of the parameters, the minimization thus effectively solves the eigenvalue problem.

As presented, the spline approximation imposes a locally polynomial structure, which is inappropriate especially close to $x=0$ and $x=1$, and particularly so in the massive case $(\gamma \neq 0)$, where the wave functions are known to behave like $\phi(x) \sim x^{\beta}$ and $\phi(x) \sim(1-x)^{\beta}$ with $\pi \beta \cot \pi \beta=1-\gamma$. It may seem, therefore, that very large values of $N$ have to be used in order to get a satisfactory solution. To avoid this unwanted feature, we also include the values of the $x_{i}$ in our set of parameters, which the minimization algorithm strives to optimize. By doing this, we allow the program to choose large interval sizes where a polynomial approximation is locally appropriate, and small sizes elsewhere. We usually increase the precision of the approximation by one order to several orders of magnitudes at fixed $N$. 4 to 5 significant digits of the eigenvalues may be obtained with as few as $N=10$ nodes.

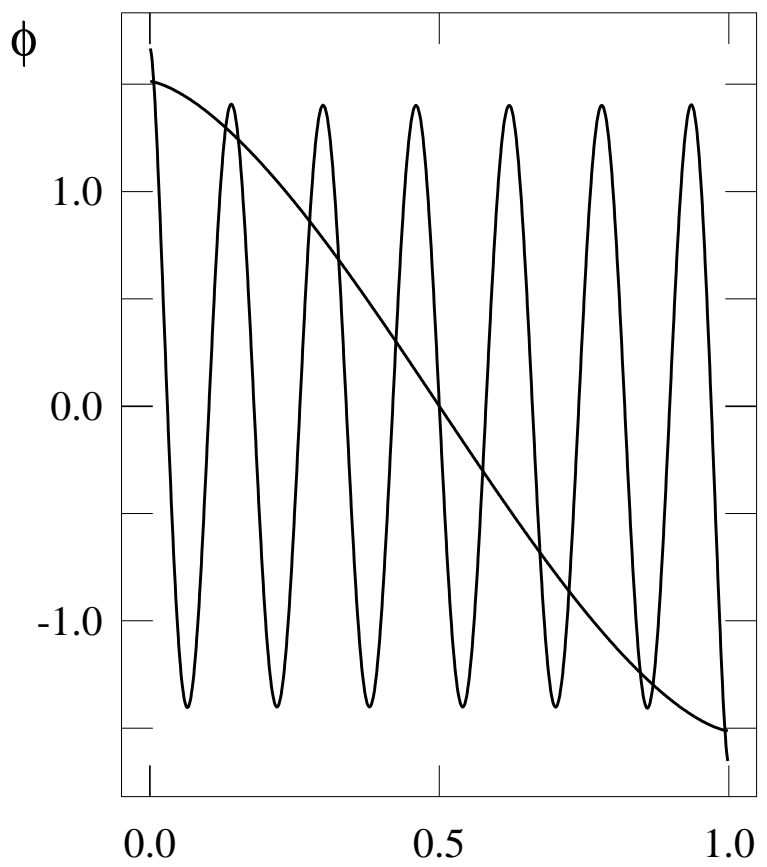

$\omega$

Figure 1: Superposed first and 13th excited state of the massless 't Hooft equation. 
In fig. (1) we give examples of the first and the 13th excited state of the massless equation (2) (all the other states may be obtained with the help of the available program). Note that the ground state is given by the analytically exact solution $\phi=1$. We observe numerically that the higher excited states states are very well described by cosines, with the exception of the endpoints $x=0$ and $x=1$ and a slight $x$-dependent phase-shift when moving away from the midpoint $x=\frac{1}{2}$.

\section{Non-Integrability}

We have computed the amplitudes for all decays involving states up to the 14th excited state and repeated this procedure for the massive case. The on-shell amplitudes, while clearly non-zero in general, display a number of features that would be interesting to explain. Some of these features differ significantly between the massless and the massive case. For example, the amplitude for the decay $\left(i, f_{1}, f_{2}\right)$ involving a highly excited state $i>>1$ going to two low-lying states $f_{1}$ and $f_{2}$ goes - for increasing $i$ - rapidly to zero in the massless case while it reaches a maximum in the massive case.

Examples of our calculations can be found in fig. (2) where we display the decay amplitude $\mathcal{A}\left(i, f_{1}, f_{2} ; \omega\right)$ vs $\omega$, for different initial and final states. In figs. (2a) and (2b) we consider symmetric decays $(i, f, f)$ in which the on-shell parameters $\omega_{ \pm}$satisfy $\omega_{+}+\omega_{-}=1$, and where the two decay amplitudes are trivially identical.

Note that all these decays are parity-allowed and that we obtain $\mathcal{A}\left(\omega_{+}\right)=\mathcal{A}\left(\omega_{-}\right)$. We have carefully checked the numerics, and clearly obtain non-zero decay amplitudes proving our claim: $Q C D_{2}$ is not integrable.

It should also be mentioned that the first few possible decay amplitudes are rather small (see e.g. (5-1-1)). Fig. (2b) (for the decay (13-3-3)) shows that some decays nevertheless have big amplitudes. This seems to be the case whenever we are close to threshold (i.e. when the momentum of the decay products is small in the rest frame of the decaying particle. Finally, we show in fig. (2c) a case of an asymmetric decay (9-3-1), in which eq. (5) gives two non-trivially related numbers for the on-shell value of $\omega$. Nevertheless, we find that the decay probabilities are equal to very high precision, illustrating again the quality of the numerical solution. Let us mention that the same features are found in the massive case, which is not further discussed here. For the parity-forbidden decays we find non-zero probabilities of equal magnitude, but opposite sign (for any value of the mass parameter 


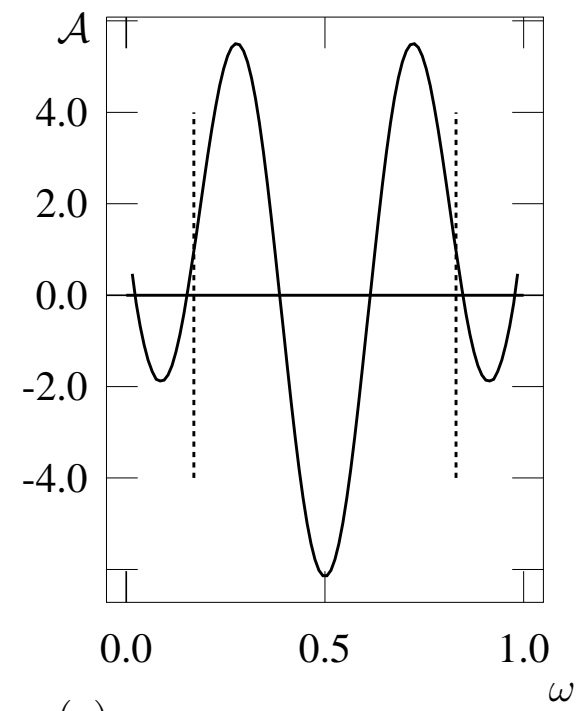

(a)

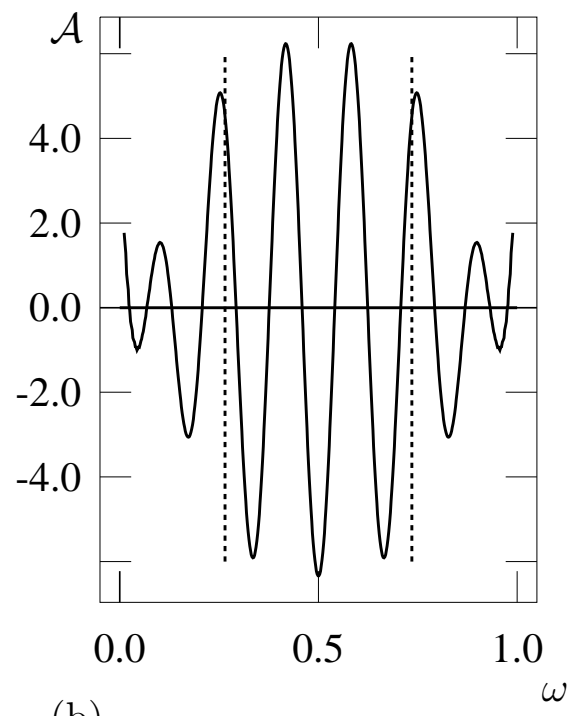

(b)

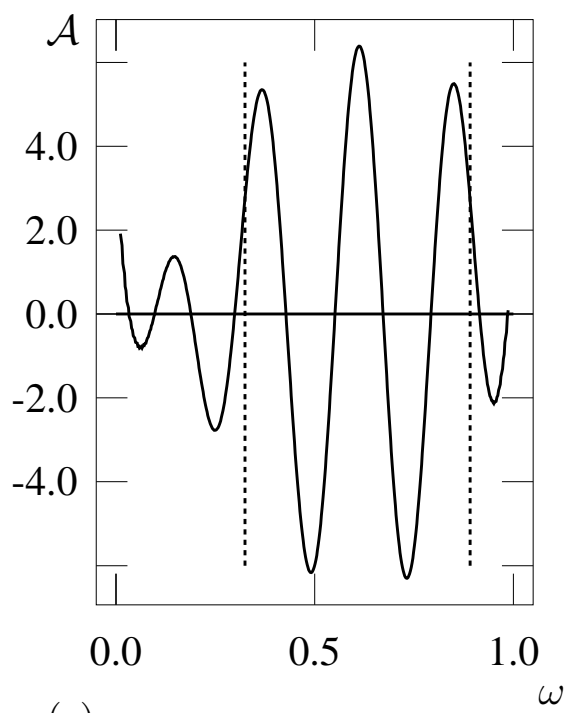

(c)

Figure 2: Amplitudes for three sample decay processes: (a) (5-1-1), (b) (13-3-3) and (c) (9-3-1). The vertical bars mark the on-shell values $\omega_{+}$(right decays) and $\omega_{-}$(left decays). 
$\gamma)$

$$
\mathcal{A}\left(i, f_{1}, f_{2} ; \omega_{+}\right)=(-1)^{1+\sigma_{i}+\sigma_{f_{1}}+\sigma_{f_{2}}} \mathcal{A}\left(i, f_{1}, f_{2} ; \omega_{-}\right),
$$

which is a convolution identity that is non-trivial except for identical final states $f_{1}=f_{2}$ (where $\omega_{+}=1-\omega_{-}$). It is expected on physical grounds: The probability for $f_{1}$ going to the right or the left has to be the same.

\section{Pion Decoupling}

An intriguing by-product of the present work is the "experimental" fact that the pions cannot be produced through the decay of massive states: $\mathcal{A}=0$ as soon as $f_{1}$ or $f_{2}$ are pions. The existence of massless pions in the 't Hooft model is a consequence of chiral $U(1)$ symmetry as shown in [11]. Although in (1) both $S U(N)$ and $U(1)$ vector symmetry are gauged, the large $N$ limit takes the $U(1)$ gauge coupling to zero in a way that suppresses the chiral $U(1)$ anomaly. The simplest argument for this is that the anomaly receives contributions from Feynman diagrams with one fermion loop, which by standard large $N$ counting is $O(1 / N)$. Conservation of the vector $U(1)$ current in two dimensions allows writing $J^{\mu}=\epsilon^{\mu \nu} \partial_{\nu} \phi$, and conservation of the chiral $U(1)$ current $J_{\mu}^{5}=\epsilon_{\mu \nu} J^{\nu}$ then implies the existence of a free massless boson [12]. Thus the pion can be considered an $N=\infty$ artifact, and the above argument gives an indirect explanation for the observed on-shell decoupling.

However, it is not at all clear in which way the decoupling manifests itself directly in the mathematical description of the 't Hooft model. The pion wave function $\phi_{\pi}$ is the only known exact solution of the chiral $(\gamma=0)$ bound state equation (2):

$$
\phi_{\pi}(x)=1 \quad \text { with } \quad \mu_{\pi}^{2}=0 \quad \text { and } \quad \Phi_{\pi}(x)=\frac{-1}{x(1-x)} .
$$

If, say, the second final state is a pion $\left(f_{2}=\pi\right)$ we thus expect the amplitude (3) to be zero for any initial state $i$ and first final state $f_{1}$ with $\mu_{i}>\mu_{f_{1}}$ :

$$
\mathcal{A}\left(\omega_{+}\right)=\int_{0}^{\omega_{+}} d x \phi_{i}(x) \phi_{f_{1}}\left(\frac{x}{\omega_{+}}\right)\left[\frac{1}{\omega_{+}-x}+\frac{1}{x-1}\right]-\frac{1}{\omega_{+}} \int_{\omega_{+}}^{1} d x \phi_{i}(x) \Phi_{f_{1}}\left(\frac{x}{\omega_{+}}\right)=0,
$$

if we choose the on-shell value (5):

$$
\omega_{+}=\frac{\mu_{f_{1}}^{2}}{\mu_{i}^{2}} .
$$

We indeed verified the validity of eq. (9) for all possible decays of the first 14 excited states, using our numerical method. Examples are shown in fig. (3a) (for the decay (5-4$0)$ ) and fig. (3b) (for the decay (13-3-0)). In the last example, the numerically computed 
on-shell value $\omega$ differs from the zero of the function $\mathcal{A}(\omega)$ by about $10^{-4}$, which is a very good precision considering the order of the initial excited state, and the small number of parameters in our spline approximation $(N=60$ was used).

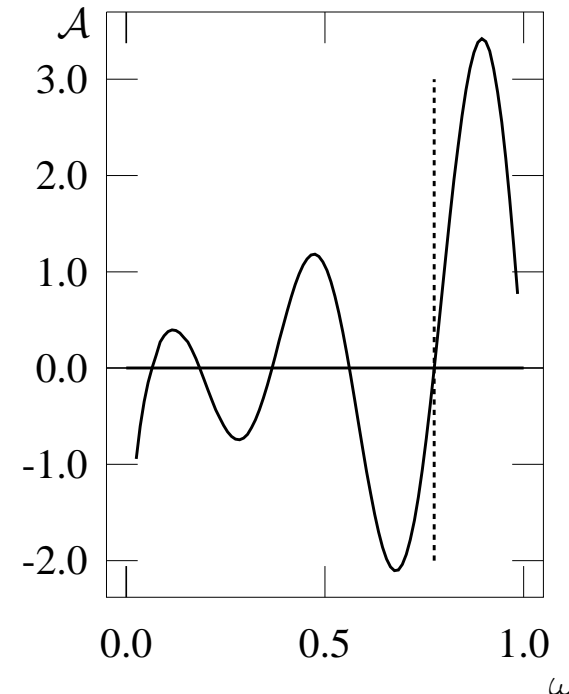

(a)

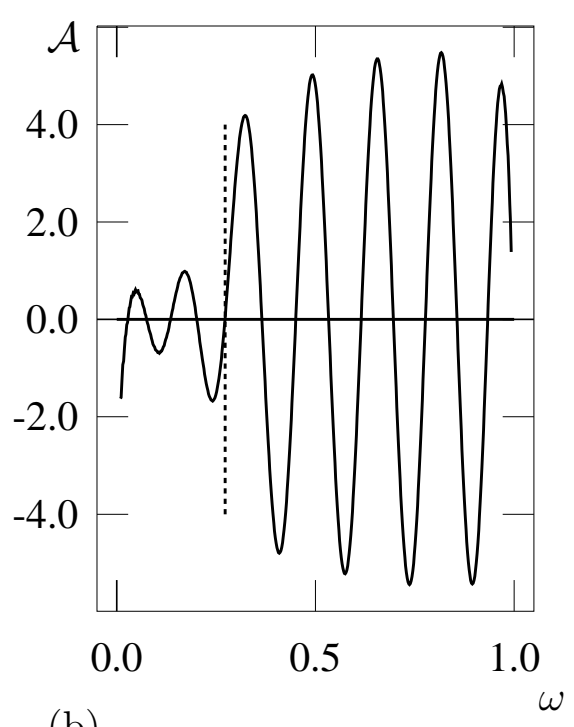

(b)

Figure 3: Amplitudes for two sample decay processes, involving pions: (a) (5-4-0) and (b) (13-4-0). The vertical bar marks the on-shell value $\omega_{+}$. It is seen that the pions decouple on-shell.

We checked that the amplitudes become non-zero as soon as the quarks acquire a mass. It is a challenging problem to give a mathematical proof of the convolution formula (9), starting from the bound state equation (2).

\section{Acknowledgements}

We would like to thank M. C. Batoni-Abdalla and E. Abdalla for collaboration in the initial stage of this project, as well as for several constructive discussions. W. K. thanks the CERN theory division for hospitality. FORTRAN programs can be obtained by e-mail (from any of the authors).

\section{References}

[1] G. 't Hooft, Nucl. Phys. B75 (1974) 461. 
[2] E. Abdalla and M. C. B. Abdalla, Int. J. Mod. Phys. A10 (1995) 1611; E. Abdalla and M. C. B. Abdalla, Phys. Lett. B337 (1994) 347; E. Abdalla, Towards a solution of two-dimensional QCD, hepth-9510139, to be published in the proceedings of ICTP Workshop on Strings, Gravity and Related Topics, Trieste, Italy, 29-30 June 1995.

[3] E. Abdalla and M. C. B. Abdalla, Updating $Q C D_{2}$, Phys. Rept. 265 (1996) 253.

[4] E. Abdalla and M. C. B. Abdalla, Phys. Rev. D52 (1995) 6660.

[5] A. B. Zamolodchikov and Al. B. Zamolodchikov, Ann. Phys. 120 (1979) 253.

[6] E. Abdalla (unpublished) pointed out to us that the integrability of $Q C D_{2}$ could be tested by computing pair-production probabilities in the 't Hooft model.

[7] J. Hanson, R. D. Peccei and M. K. Prasad, Nucl. Phys. B121 (1977) 477; R. L. Jaffe and P. F. Mende, Nucl. Phys. B369 (1992) 189.

[8] C. G. Callan Jr., N. Coote, and D. J. Gross, Phys. Rev. D13 (1976) 1649.

[9] J. Stoer and R. Bulirsch, Introduction to Numerical Analysis, Springer Verlag, New York (1980).

[10] W. H. Press, S. A. Teukolsky, W. T. Vetterling, B. P. Flannery, Numerical Recipes, 2nd edition, Cambridge University Press (1992).

[11] I. Affleck, Nucl. Phys. B265 [FS15] (1986) 448.

[12] E. Witten, Nucl. Phys. B145 (1978) 110. 